\title{
FAILURE OF SLIDING NAIL-PLATE FIXATION IN SUBCAPITAL FRACTURES OF THE FEMORAL NECK
}

\author{
T. I. S. BROWN, C. COURT-BROWN
}

\author{
From Princess Margaret Rose Orthopaedic Hospital, Edinburgh
}

\begin{abstract}
A retrospective study of $\mathbf{2 0 0}$ cases of subcapital fracture of the neck of the femur treated by sliding nail-plate fixation has been carried out. Failure of fixation within three months occurred in forty-two cases (21 per cent). These failures could, in part, be attributed to a combination of the severity of the fracture and various imperfections in technique. The age of the patient and the angle of the nail-plate had no significant effect on the result.
\end{abstract}

Controversy surrounding the intracapsular fracture of the femoral neck - " the unsolved fracture" (Dickson 1953) - has continued unabated since 1883 when Senn stated: "The only cause for the non-union in the case of intracapsular fracture is to be found in our inability to maintain perfect coaption and immobilisation of the fragments during the time required for bony union to take place." The optimal method of achieving this "perfect coaption and immobilisation" is still being sought, and the fact that no single universally accepted device exists is reflected in the plethora of different fixation materials, a recent review (Tronzo 1974) listing seventy-six. Individual orthopaedic units can only have had experience of a small number of different devices, but since various authors expressed dissatisfaction with the results obtained using the single Smith-Petersen nail (Küntscher 1953; Garden 1961; Brown and Abrami 1964; McQuillan, Abernethy and Guy 1973; Barnes et al. 1976) sliding nail and plate combinations have become popular.

The principle of a sliding type of fixation was introduced in the 1940 s but the first sliding device to gain popularity was the Pugh nail (Pugh 1955), and since then various authors have reported encouraging results with similar devices (Brown and Abrami 1964; Barr 1973; Fielding 1975; Frandsen and Jorgensen 1977). On this basis the Howmedica sliding nail and plate combination was adopted by a number of surgeons in the Royal Infirmary of Edinburgh in 1974. After three years of use, there was a subjective impression of a high failure rate and accordingly this study was undertaken to evaluate the cases so treated. The findings in 200 patients with subcapital fractures of the femoral neck fixed internally with sliding nail and plate are presented. The study was limited to a follow-up period of three months, accepting that the majority of fractures would not have united by this time; if the sliding nail was still maintaining the head in its reduced position after three months of attempted weight-bearing, then it was at least fulfilling its purpose of internal fixation. The long-term incidence of non-union and avascular necrosis is outwith the confines of this study.

\section{MATERIALS AND METHODS}

This study involved all patients with intracapsular fractures treated by sliding nail and plate fixation in the Royal Infirmary of Edinburgh between October 1974 and December 1977. During this period around 600 patients were admitted with intracapsular fractures, but many were treated by other methods which included total hip arthroplasty, primary hemiarthroplasty, single pin, double divergent pins and Moore's pins. The choice of fixation was determined by the severity of the fracture, the age of the patient, and the individual preference of the surgeon-in-charge, there being seven different consultants involved. The fractures were graded according to Garden's (1961) classification: Grade I-incomplete fracture (impacted valgus fracture); Grade II-complete fracture without displacement; Grade III-complete fracture with partial displacement; Grade IVcomplete fracture with full displacement.

In general, Grade I or II fractures were treated with a single pin. Primary hemiarthroplasty was often advocated for Grade IV fractures in the very elderly group, while fractures in the presence of rheumatoid disease were considered for total hip replacement. Two hundred and fifty-two patients were treated with sliding nail and plate, the majority having Grade III and IV fractures. Thirteen ( 5.2 per cent) have been lost to follow-up; thirty-three (13.1 per cent) died within the three-month period; six (2.4 per cent) did not have adequate radiographs. Thus 200 patients were studied, 171 women and twenty-nine men, the age range being from forty-three to ninety-five years.

Adequate radiographs were required initially for the grading of the fracture, during the operation for the criteria of reduction, and serially up to three months later. As the patients had been operated on by many junior surgeons, absolute standardisation of method of reduction, type of anaesthesia, and operative technique was impossible. However, in all cases the delay between presentation and operation was kept to a minimum, this delay being dictated by the patient's medical state and the availability of operating time, while early weight-bearing was encouraged.

The patients were studied for a period of three months. Failure of fixation was recorded when the patient had clinical and radiological

T. I. S. Brown, F.R.C.S., 25 Peel Street, Christchurch 5, New Zealand.

C. Court-Brown, M.B., Ch.B., Registrar, Department of Surgery, Bangour General Hospital, Broxburn. West Lothian EH52 6LW. Scotland. Requests for reprints should be sent to Mr C. Court-Brown. 

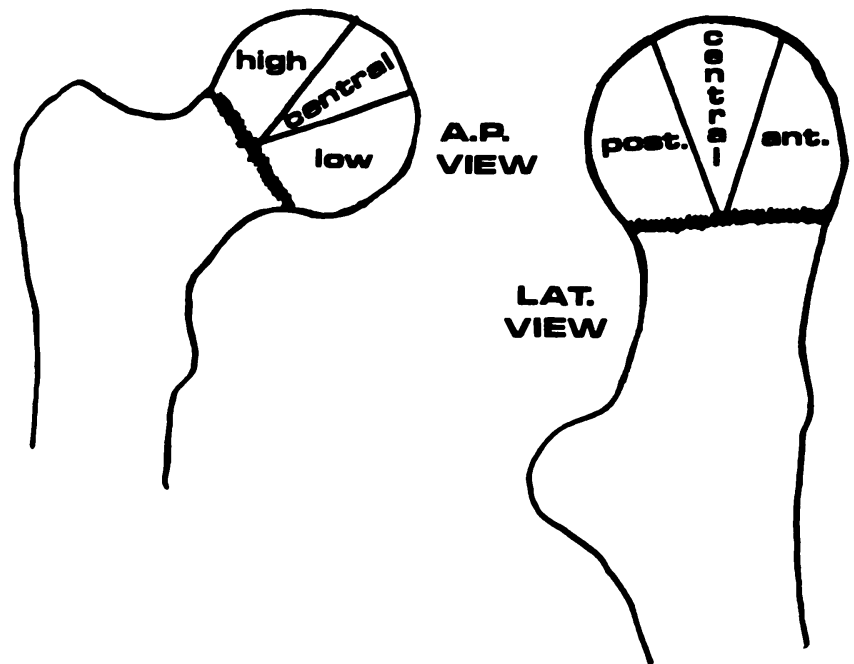

Fig. 1

Anteroposterior and lateral planes of the femoral head divided into sectors.

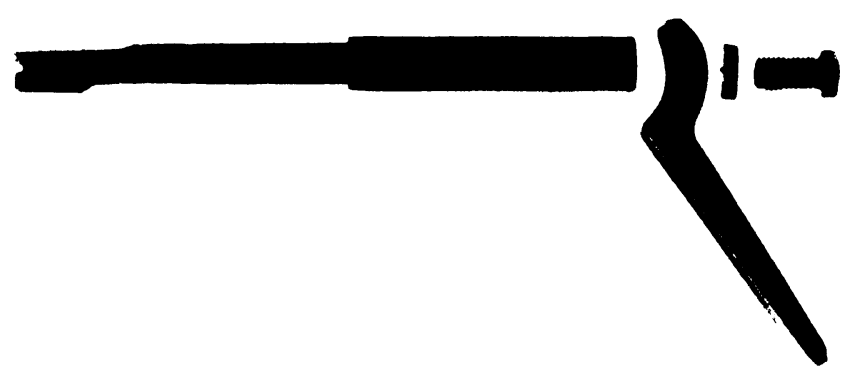

Fig. 2

The sliding nail fully extended and showing the variable-angle side plate. loss of the reduced position of the femoral head to such an extent that further operation was indicated. Assessment was made of the patient's age, the presence or absence of biochemical osteomalacia, the grading of the fracture in accordance with Garden's classification (Garden 1961), the accuracy of reduction using Garden's alignment index (Garden 1961), the nail-plate angle, the position of the nail in the head of the femur as seen on standard anteroposterior and lateral radiographs - these positions being divided into the sectors illustrated in Figure 1-and the distance between the tip of the nail and the articular surface of the femoral head.

The sliding nail and plate which was used is illustrated in Figure 2. Made of Vitallium, the device consists of an outside barrel or sleeve containing an inner extensible nail with a maximal length of 7.3 centimetres. The distal 1.5 centimetres is trifin in shape to cut into and grip the cancellous bone of the femoral head. A tongue and groove arrangement in the barrel prevents rotation. The plate is connected to the nail by a self-locking washer and a bolt. The angle of insertion can vary.

\section{RESULTS}

Of the 200 patients in this study, forty-two ( 21 per cent) had failure of fixation within three months. Their further treatment is summarised in Table I. In an attempt to find reasons for this failure rate, the objective measurements mentioned previously were examined.

Age of patient. Figure 3 shows the proportion of successes and failures with age and it can be seen that age was not a significant factor $(P>0.5)$.

Grade of fracture. Comparison of the Garden classification of the fracture with failure of fixation showed a higher failure rate in Grade IV fractures (Table II). This finding was statistically significant $(P<0.01)$ and in keeping with the generally held opinion that Grade IV fractures are the most difficult group in which to obtain an accurate reduction and stable fixation.

Nail-plate angle. In the past. importance has been attached to the angle of nail and plate. Most sliding

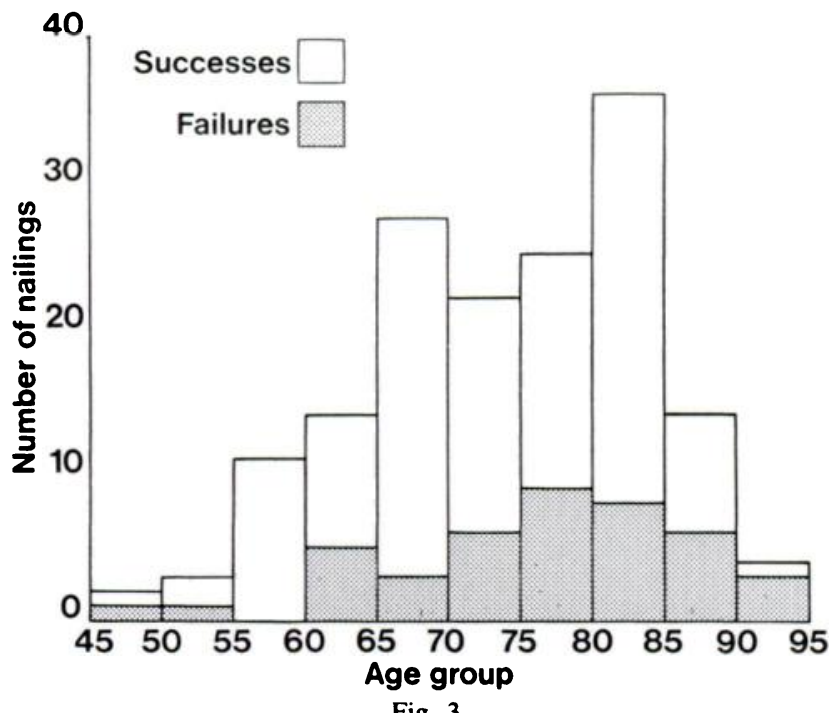

Fig. 3

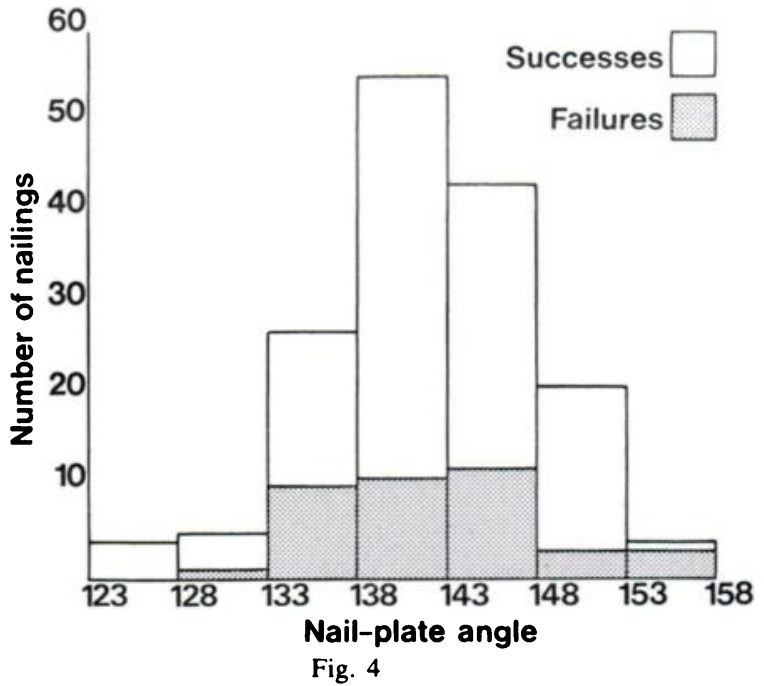

Fig. 4

Figure 3-Histogram of the nailing successes and failures in the different age groups. There is a trend to more failures in the oldest group, but numbers are too low for statistical significance. Figure 4- Histogram of nailing successes and failures with the different nail-plate angles. arranged in 5-degree bands spread around the theoretically important angles of 135 and 1.50 degrees. The numbers in the group between 1.53 and 158 degrees are too low for statistical significance to apply. 
devices have had a fixed plate (Pugh 1955; Massie 1958; Brown and Abrami 1964). However, the angles chosen have been different. Pugh and subsequently Brown designed their devices with an angle of 135 degrees, which approximated to the normal neck-shaft angle of the femur; Massie preferred a more steeply placed nail with a nail-plate angle of 150 degrees; and more recently Frandsen and Jorgensen (1977) advocated

Table I. Further treatment of forty-two cases of failed fixation

\begin{tabular}{|l|c|}
\hline \multicolumn{1}{|c|}{ Further treatment } & $\begin{array}{c}\text { Number of } \\
\text { patients }\end{array}$ \\
\hline Removal of nail only & 1 \\
Girdlestone arthroplasty & 2 \\
Moore's hemiarthroplasty & 29 \\
Thompson's hemiarthroplasty & 1 \\
Total hip replacement & 2 \\
No further procedure & 7 \\
(medically unfit) & - \\
\end{tabular}

placing the nail at as steep an angle as possible, more in line with the medial trabeculae of the head, which Backman (1957) considered to be the main direction of loading forces. It was therefore of interest to compare the various nail-plate angles with success or failure. As can be seen from Figure 4 there is no obvious difference in the groups $(P>0.1)$.

Table II. Comparison of failure of fixation with Garden grade of fracture

\begin{tabular}{|c|c|c|c|}
\hline \multirow{2}{*}{ Garden grade } & \multirow{2}{*}{$\begin{array}{c}\text { Number of } \\
\text { cases }\end{array}$} & \multicolumn{2}{|c|}{ Failures } \\
\cline { 3 - 4 } & Number & Per cent \\
\hline I & 8 & 0 & 0 \\
II & 27 & 3 & 11.1 \\
III & 35 & 5 & 14.2 \\
IV & 130 & 34 & 26.1 \\
\hline
\end{tabular}

Penetration of the nail. The distance between the end of the nail and the articular surface of the head was measured in all 200 cases on the anteroposterior radiograph. Six millimetres was taken as an arbitrary level of division and the cases were allocated to three separate groups (Table III). The trend was to a higher failure rate when the end of the nail was more than 12 millimetres from the femoral articular surface $(P<0.1)$. Position of the nail. Figure 1 shows how the position of the nail in the head was graded. The position was defined "acceptable" if the nail was either central in both views, low and posterior, or a combination of both. "Unacceptable" position was when the nail was outwith these sectors in either plane. Of the forty-two failures of fixation, thirty-two had an acceptable nail position and ten were unacceptable, giving an unacceptable rate of nail placement of 23.8 per cent.

Pattern of failure. An attempt has been made to analyse the serial radiographs of the failures and identify any pattern. Six of the forty-two failures, although demonstrated to have failed, did not have radiographs of sufficient quality for accurate measurement against Garden's alignment index, and therefore this information was recorded for only thirty-six of the failures.

The alignment index described by Garden in 1961 relates to the angle between the mainstream of trabeculae in the medial part of the femoral head and a line drawn parallel to the medial cortex of the shaft on the anteroposterior radiograph, and equates this measurement to the angle between the central axis of the head and the central axis of the neck on the lateral film.

Table III. Comparison of failure of fixation with penetration distance of nail

\begin{tabular}{|l|c|c|c|}
\hline \multirow{2}{*}{$\begin{array}{c}\text { Distance of nail } \\
\text { from } \\
\text { articular surface } \\
\text { (millimetres) }\end{array}$} & \multirow{2}{*}{$\begin{array}{c}\text { Number of } \\
\text { cases }\end{array}$} & \multicolumn{2}{|c|}{ Failures } \\
\cline { 3 - 4 } & Number & Per cent \\
\hline Under 6 & 75 & 13 & 17.3 \\
$6-12$ & 84 & 15 & 17.8 \\
Over 12 & 41 & 14 & 34.0 \\
\hline
\end{tabular}

The normal ratio is $160 / 180$ but Garden considered that an index point in the range $155 / 180$ in both planes was within the limits of acceptability (Garden 1974). The figures from the survey by Barnes et al. (1976) generally agree with this view.

In our series, eleven of thirty-six cases had an unacceptable reduction according to the alignment index, a rate of 30.5 per cent. Careful scrutiny of the serial radiographs of the failures suggested that the nail had either "cut out" or retracted prematurely, although in some cases it was impossible to state which occurrence had come first. Of the eleven cases with an unacceptable reduction, five had an unacceptable placement of the nail and in all five the mechanism of failure was "cutting out" of the nail; four with acceptable nail placement "cut out"; in the remaining two, the nail appeared to retract prematurely.

Premature retraction of the nail appeared to be the mechanism of failure in sixteen cases out of forty-two (38 per cent). Figures 5, 6 and 7 show gradual retraction with eventual complete loss of fixation so that the head falls off. In one case, despite good reduction and nailing. 
the nail failed to retract and penetrated through the head into the acetabulum. This is seen in Figures 8, 9 and 10.

Of the thirty-six failed cases which were fully documented only 30 per cent satisfied the criteria when reduction of the fracture, nail position and nail penetration were considered together.

Osteomalacia. Only one case of biochemical osteomalacia was found in the group of failures, and this would therefore not seem to be a factor of importance.

\section{DISCUSSION}

The essential fact that emerged was that 21 per cent of our 200 cases had a failure of fixation within three months. On closely studying these failures, we have found in the majority of cases evidence of unsatisfactory reduction and of unsatisfactory techniques in using the sliding nail device.

We are not alone in admitting failure with this device. Brown and Abrami (1964) reported a 21.2 per cent failure rate in a series of 146. Barr (1973) had a 6.6 per cent failure rate in a series of seventy-five patients, in which fifteen of the fractures were Garden Grade II. Frandsen and Jorgensen (1977), using the same device, had a 22 per cent failure rate in their series of forty-six patients. Apart from reporting the statistics, no critical study has been made of these failures.

Numerous authors have emphasised the importance of perfect reduction and secure fixation (Garden

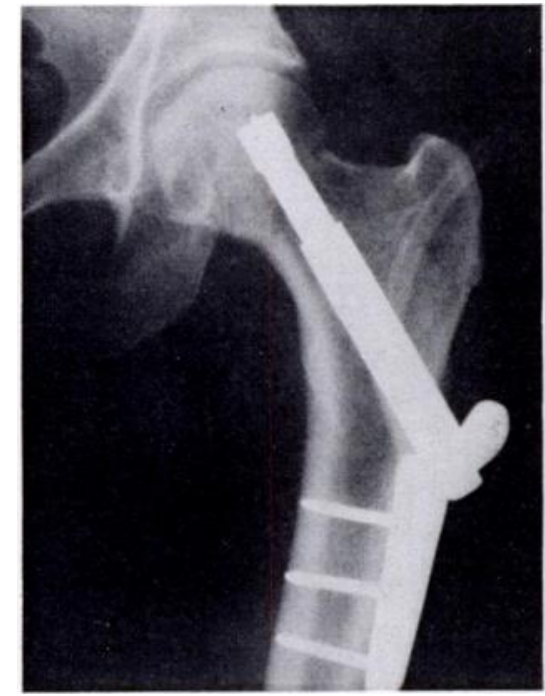

Fig. 5

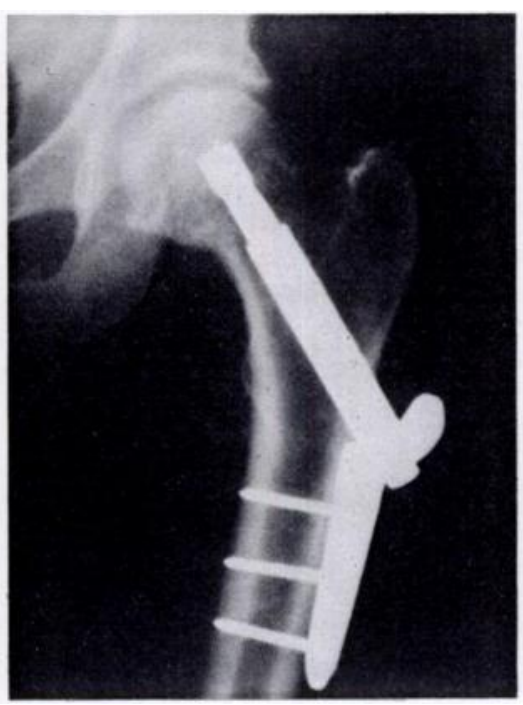

Fig. 6

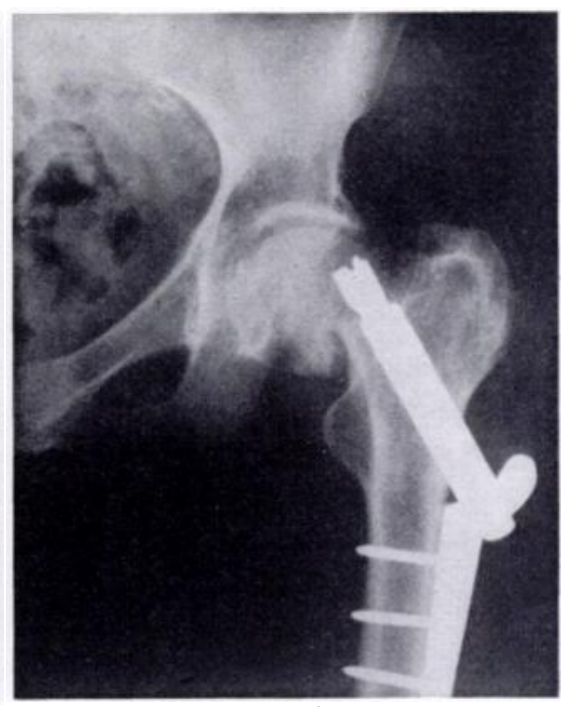

Fig. 7

Serial radiographs showing gradual retraction of the nail from the position immediately after operation to failure within three months.

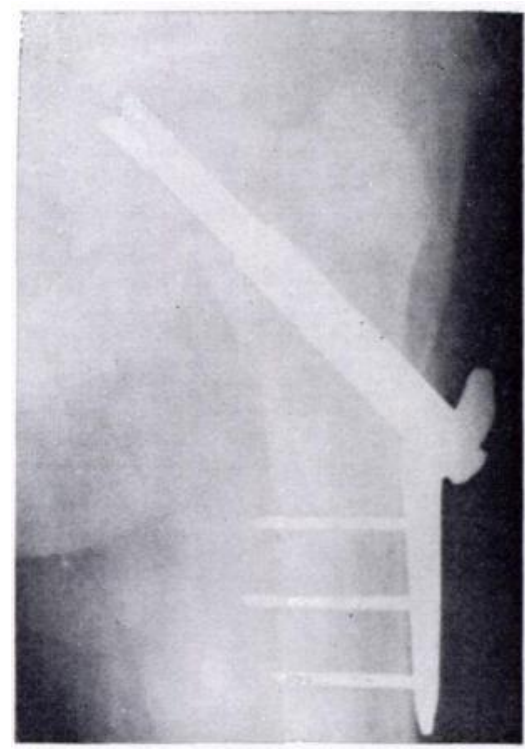

Fig. 8

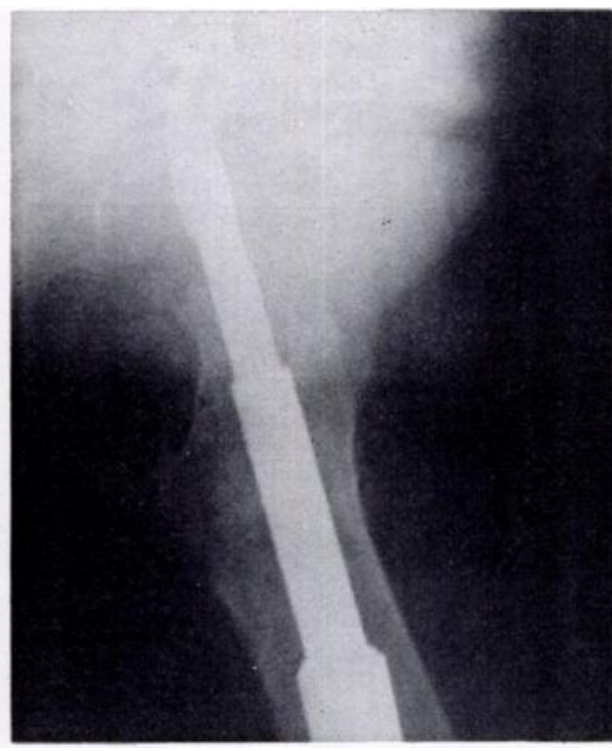

Fig. 9

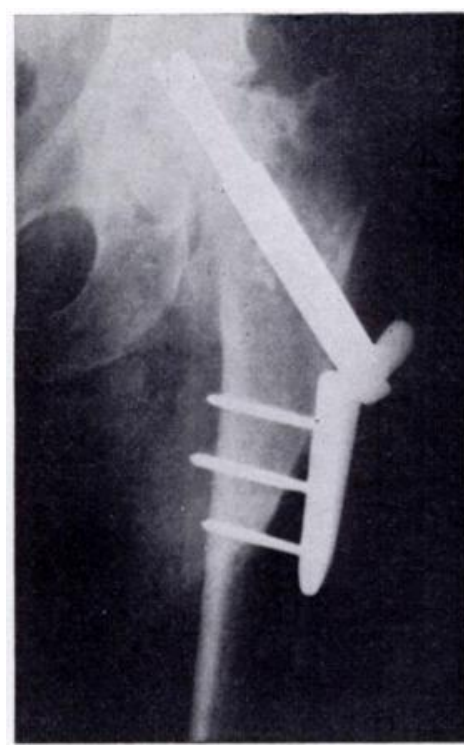

Fig. 10

Serial radiographs showing acceptable reduction, nail placement and penetration, but with the nail failing to retract and penetrating the acetabulum. 
1961; Barnes et al. 1976). A realistic fact in the treatment of displaced subcapital fractures is that reduction is probably never "perfect" and to achieve even an "acceptable" position is often difficult (Cleveland and Fielding 1954). In theory the perfect nailing should be easier to achieve, but first of all the perfect nail must be defined. Our results indicated that the angle of the nail in the neck was not of critical importance. What did seem to be important, however, was the placement of the nail in the head of the femur; the upper and anterior segments of the head should be avoided. Considering the length of the nail, it is logical in mechanical terms to obtain maximal advantage by having the tip of the nail as far across the site of the fracture as possible, without penetrating the femoral articular surface.

However, all these criteria are merely measurements made on radiographs and do not take into account the effect of the grip obtained by the nail in the head, nor the effect of weight-bearing or torque forces upon this grip. In our series, despite satisfying all the accepted criteria for adequate reduction and nailing, we could find no obvious cause in 30 per cent of our failures. Three cases in this group were Garden Grade II fractures, which are generally accepted to have a much better prognosis on account of inherent stability, and this finding would seem to cast serious doubts on the ability of this sliding device to maintain its grip in the femoral head. In many of the cases, early retraction of the nail appeared to be the mode of failure, and this retraction could only have occurred with the trifin tip of the nail loosening its purchase on the cancellous bone of the head.

Impaction of the fracture surfaces is advocated by many workers, as it increases the stability at the site of the fracture, but although this proposal seems logical, it is not something that can be measured objectively.

Early weight-bearing was encouraged in our series and this may have had some influence on loosening of the nail. Probably the best results in the use of a sliding device in intracapsular displaced fractures were those reported by Fielding, Wilson and Ratzan (1974), who claimed there was no early retraction of the nail in their series; but their patients did not bear full weight on the affected limb until union had been established. Again, in a series of intracapsular fractures treated with a sliding nail, Nieminen (1975) found slightly better results than ours in early weight-bearers and stated that bony union was relatively more frequent and failures relatively less frequent in this group. Certainly, with regard to the patient's general health, the advantages of early weight-bearing far outweigh the disadvantages.

We have found that this operation demands a precision of technique which has not been emphasised sufficiently, and that in general junior surgeons of limited experience are usually assigned to this task. In practice, reductions will continue to be imperfect, but fixation of the femoral head in the best attainable position, and holding it there until union occurs, is the prime objective of the operation. In our series, the failure rate is unacceptably high and further evaluation of other techniques is indicated.

We would like to thank our orthopaedic consultants for permission to study their cases, and Mr M. Devlin for photographic assistance.

\section{REFERENCES}

Backman, S. (1957) The proximal end of the femur. Acta Radiologica, Supplement 146.

Barnes, R., Brown, J. T., Garden, R. S., and Nicoll, E. A. (1976) Subcapital fractures of the femur-A prospective review. Journal of Bone and Joint Surgery, 58-B, 2-24.

Barr, J. S., Jun. (1973) Experiences with a sliding nail in femoral neck fractures. Clinical Orthopaedics and Related Research, 92, 63-68.

Brown, J. T., and Abrami, G. (1964) Transcervical femoral fracture. Journal of Bone and Joint Surgery, 46-B, 648-663.

Cleveland, M., and Fielding, J. W. (1954) A continuing end-result study of intracapsular fracture of the neck of the femur. Journal of Bone and Joint Surgery. 36-A, 1020-1030.

Dickson, J. A. (1953) The "unsolved" fracture. Journal of Bone and Joint Surgery, 35-A, 805-822.

Flelding, J. W. (1975) Pugh nail fixation of displaced femoral neck fractures. Clinical Orthopaedics and Related Research, 106, $107-116$.

Fielding, J. W., Wilson, S. A., and Ratzan, S. (1974) A continuing end-result of displaced intracapsular fractures of the neck of the femur, treated with the Pugh nail. Journal of Bone and Joint Surgery, 56-A, 1464-1472.

Frandsen, P. A., and Jorgensen, F. (1977) Osteosynthesis of medial fractures of the femoral neck by sliding nail-plate fixation. Acta Orthopaedica Scandinavica, 48, 57-62.

Garden, R. S. (1961) Low-angle fixation in fractures of the femoral neck. Journal of Bone and Joint Surgery, $43-B, 647-663$.

Garden, R. S. (1974) Reduction and fixation of subcapital fractures of the femur. Orthopaedic Clinics of North America, 5, 683-712.

Küntscher, G. (1953) Die vollautomatische Schenkelhalsnagelung. Zeitschrifi für Orthopädie und ihre Grenzgebiete, 84, 17-28.

Massie, W. K. (1958) Functional fixation of femoral neck fractures: telescoping nail technic. Clinical Orthopaedics, 12 , $230-255$.

McQuillan, W. M., Abernethy, P. J., and Guy, J. G. (1973) Subcapital fractures of the neck of the femur treated by double-divergent fixation. British Journal of Surgery, 60, 859-866.

Nieminen, S. (1975) Early weightbearing after classical internal fixation of medial fractures of the femoral neck. Acta Orthopaedica Scandinavica, 46, 782-794.

Pugh, W. L. (1955) A self-adjusting nail-plate for fractures about the hip joint. Journal of Bone and Joint Surgery, $37-\mathbf{A}, 1085-1093$.

Senn, N. (1883) Fractures of the neck of the femur with special reference to bony union after intra-capsular fracture. Transactions of the American Surgical Association, 1, 333-441.

Tronzo, R. G. (1974) Hip nails for all occasions. Orthopaedic Clinics of North America, 5, 479-491. 